\title{
Etapas electrónicas de potencia resonantes multi-salida para calentamiento por inducción doméstico.
}

\author{
Pablo Guillén, Héctor Sarnago, Óscar Lucía y José Miguel Burdío \\ Grupo de Electrónica de Potencia y Microelectrónica (GEPM) \\ Instituto de Investigación en Ingeniería de Aragón (I3A) \\ Universidad de Zaragoza, Mariano Esquillor s/n, 50018, Zaragoza, Spain. \\ Tel.+34-976762707, e-mail: pquillenm@unizar.es
}

\section{Resumen}

En este artículo se propone una etapa inversora matricial multi-salida controlada mediante una estrategia de modulación asimétrica para su utilización en cocinas de inducción de superficie flexible. De esta forma se consigue una implementación con un número reducido de dispositivos y que ofrece un control de potencia versátil y preciso.

\section{Introducción}

Las cocinas de inducción flexibles, que logran mayor libertad en la selección del tamaño, forma y posición del recipiente, basan su funcionamiento en estructuras multi-inductor (Fig. 1). Las etapas electrónicas utilizadas típicamente para transmitir potencia a estas estructuras presentan implementaciones con una gran cantidad de dispositivos de potencia [1], resultando costosas y complejas. Por tanto, resulta interesante abordar el diseño de inversores multi-salida que permitan reducir el número de componentes electrónicos necesarios para el funcionamiento de la cocina. Entre estos, las estructuras matriciales [2] se presentan como una alternativa recomendable ya que añaden mejoras notables de cara al usuario final como una detección de recipiente más sencilla [3] o un control de potencia más rápido y preciso [4].

\section{Inversor matricial ZVS}

La etapa multi-salida propuesta (Fig. 2) es una versión unidimiensional del inversor resonante matricial ZVS [5] y, por lo tanto, presenta un único transistor de lado alto, $\mathrm{S}_{\mathrm{H}}$, común para todas las ramas de salida. Cada una de estas ramas está compuesta por un diodo serie, $\mathrm{D}_{\mathrm{s}, \mathrm{i}}$, un transistor de lado bajo, $\mathrm{S}_{\mathrm{L}, \mathrm{i}}$, con un diodo en antiparalelo, $\mathrm{D}_{\mathrm{L}, \mathrm{i}}$, un diodo en antiparalelo de lado alto, $\mathrm{D}_{\mathrm{H}, \mathrm{i}}$, y la carga de inducción. Estas cargas son modeladas por su resistencia, $R_{\mathrm{L}, \mathrm{i}}$, e inductancia, $L_{\mathrm{r}, \mathrm{i}}$, equivalentes.
Los parámetros disponibles para el control de potencia en las ramas activas se presentan en la Fig. 3. El control de la frecuencia de conmutación, $f_{s w}, \mathrm{y}$ el ciclo de servicio, $D$, son comunes a todos los inductores activos, estableciendo su potencia máxima. De esta forma, mediante la activación no complementaria del transistor inferior de cada una de las cargas, es decir, incrementando el ángulo de disparo, $\alpha_{\mathrm{i}}$, se consigue ajustar la potencia transmitida a cada inductor (Fig. 4) [6].

La desactivación de las distintas cargas se consigue gracias a la presencia del diodo serie que fija la tensión del condensador de resonancia, $C_{\mathrm{r}}$, cuando el transistor inferior de la rama no es activado.

\section{Prototipo experimental}

Con la finalidad de probar experimentalmente la etapa y la estrategia de modulación, se diseña y construye un prototipo con 12 salidas y que permite la alimentación desde hasta dos fases de red (Fig. 5).

El prototipo está constituido por dos PCB de potencia interconectadas mediante una tercera PCB que incluye el control y las medidas. Debido al número de dispositivos semiconductores que requieren refrigeración, se opta por una implementación de la potencia en PCB con substrato de aluminio (IMS).

El prototipo ha sido validado en aplicación real teniendo en cuenta tanto el diseño electrónico como la selección de componentes y el dimensionamiento del sistema de refrigeración. En la Fig. 6 se muestran las formas de onda tanto de las señales de control de los transistores como de la tensión aplicada a la carga y la corrietne por la misma al operar con la estrategia de modulación propuesta.

\section{Conclusiones}

El inversor multi-salida propuesto permite reducir el número de dispositivos de potencia necesarios $\mathrm{y}$ presenta una implementación completa mediante 
dispositivos semiconductores que incrementa la fiabilidad del sistema. Se trata de la mayor reducción de dispositivos en este tipo de estructuras lograda hasta la fecha.

La estrategia de modulación asimétrica consigue el control individual de potencia en cargas activas simultáneamente con distintos parámetros equivalentes, operando de forma continua y con una frecuencia de conmutación común. Se consigue, por lo tanto, unas prestaciones en control muy superiores a las existentes en las etapas actuales.

Por último, el diseño y construcción de un prototipo funcional para su integración en una cocina comercial ha servido para la validación de la etapa electrónica y la estrategia de modulación propuestas, comprobando su versatilidad y su alta eficiencia.

\section{REFERENCIAS}

[1] P. Guillen, H. Sarnago, L. Ó, and J. M. Burdío, "MultiOutput Resonant Power Converters for Domestic Induction

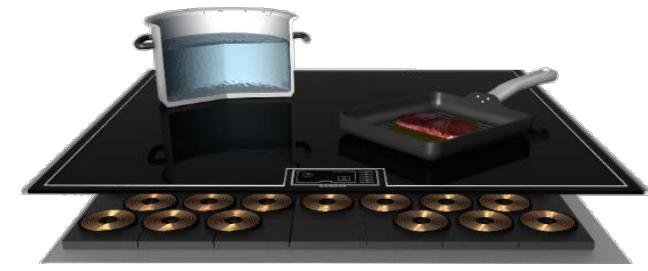

Fig. 1. Cocina de inducción flexible.

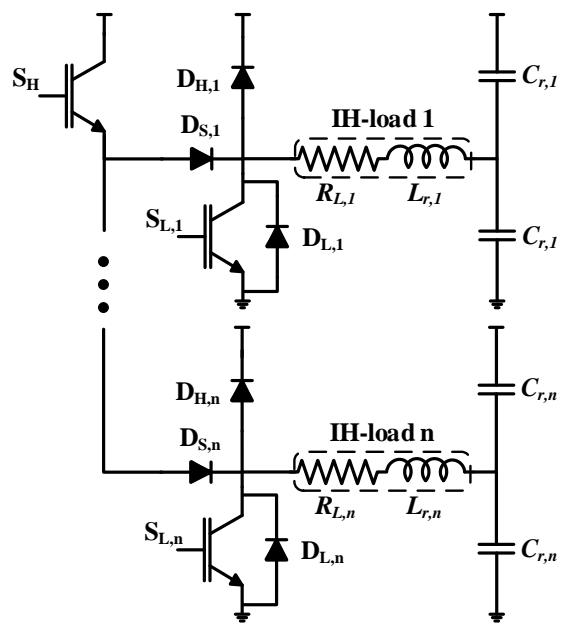

Fig. 2. Inversor resonante matricial multi-salida.

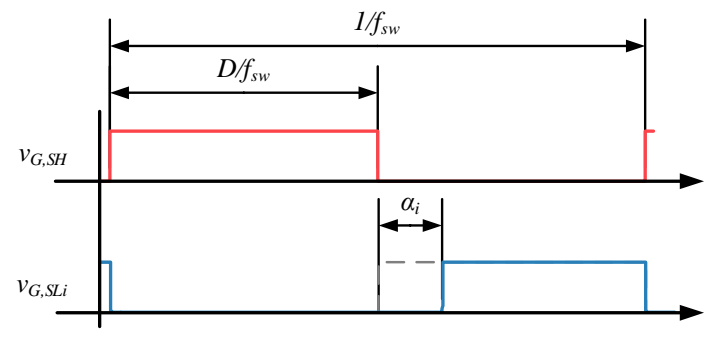

Fig. 3. Parámetros de control del inversor multi-salida.
Heating," in IECON 2020 The 46th Annual Conference of the IEEE Industrial Electronics Society, 2020, pp. 43204327.

[2] H. Sarnago, O. Lucia, and J. M. Burdío, "Multiple-output ZCS resonant inverter for multi-coil induction heating appliances," in 2017 IEEE Applied Power Electronics Conference and Exposition (APEC), 2017, pp. 2234-2238.

[3] H. Sarnago, L. Ó, and J. M. Burdío, "FPGA-Based Resonant Load Identification Technique for Flexible Induction Heating Appliances," IEEE Transactions on Industrial Electronics, vol. 65, pp. 9421-9428, 2018.

[4] P. Guillen, H. Sarnago, O. Lucia, and J. M. Burdio, "MainsSynchronized Pulse Density Modulation Strategy Applied to a ZVS Resonant Matrix Inverter," IEEE Transactions on Industrial Electronics, pp. 1-1, 2020.

[5] H. Sarnago, P. Guillén, J. M. Burdío, and O. Lucia, "Multiple-Output ZVS Resonant Inverter Architecture for Flexible Induction Heating Appliances," IEEE Access, vol. 7, pp. 157046-157056, 2019.

[6] P. Guillén, H. Sarnago, O. Lucía, and J. M. Burdío, "Asymmetrical Non-Complementary Modulation Strategies for Independent Power Control in Multi-Output Resonant Inverters," IEEE Journal of Emerging and Selected Topics in Power Electronics, pp. 1-1, 2020.

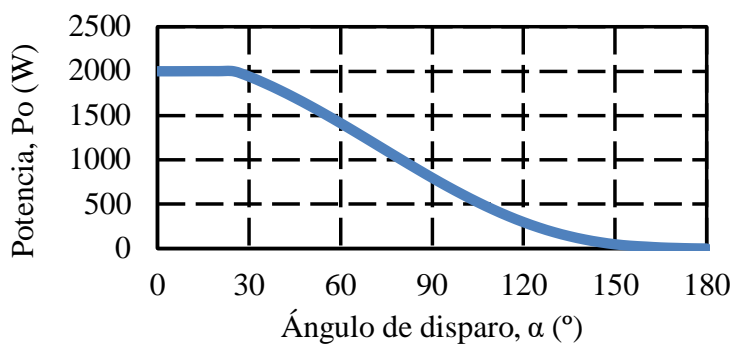

Fig. 4. Potencia entregada en función del parámetro de control $\alpha_{\mathrm{i}}$.

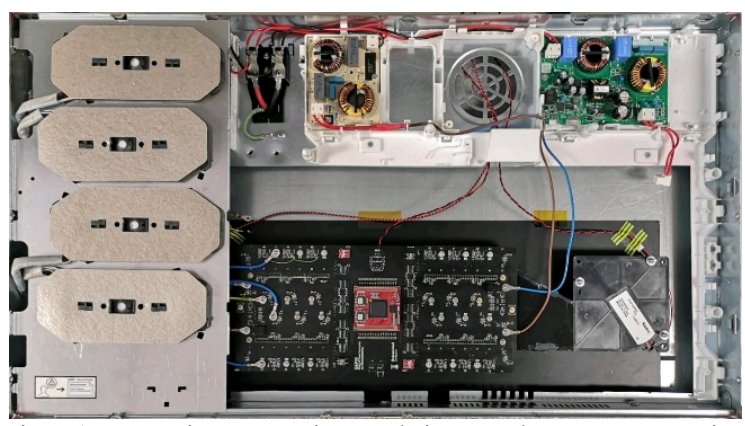

Fig. 5. Prototipo experimental integrado en una cocina comercial.

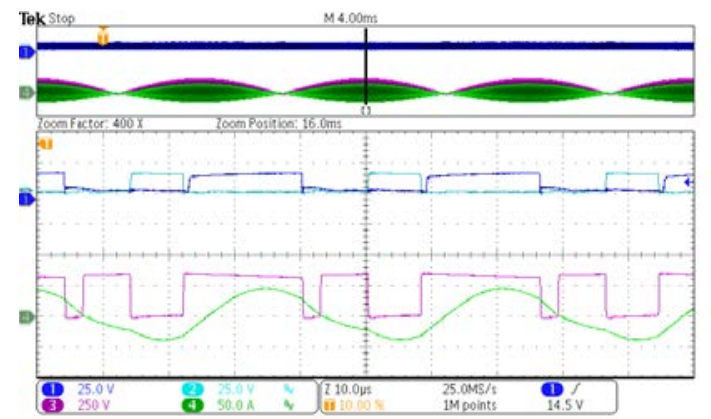

Fig. 6. Formas de onde experimentales para una potencia de salida de $1000 \mathrm{~W}$.

Revista “Jornada de Jóvenes Investigadores del I3A”, vol. 8 (Actas de la IX Jornada de Jóvenes Investigadores del I3A -11 de diciembre de 2020). ISSN 2341-4790. 